\title{
76. Cerebral Aneurysm. Report of 36 Cases
}

\author{
Shuro Nishimura, Takashi Nakamura, Tamotsu Asakura, \\ Yoshihiko Umebayashi, Kunihiko Osaka and Kazuo Ebina \\ Neurosurgery, Kitano Hospital, Tazuke Kofukai Research Institute, Osaka
}

Analysis of 36 cases of cerebral aneurysm is reported.

1) Their location and numbers are as follows: Internal carotid 13, anterior cerebral 6 , anterior communicating 12 , middle cerebral 4 , and posterior communicating 1 .

2) Surgical treatment was performed in 22 cases and 9 died after surgery. Operation was not made in 14 patients and 11 out of them died.

3) Operative techniques performed are as follows: Clipping in 4 (2 died), trapping in 5 ( 3 died), coating in 8 (no death), evacuation of hematoma in 2 ( 2 died) and carotid ligation in 3 ( 2 died). Coating with EDH adhesive is the best among those measures, regarding operative result.

4) Nine cases, in which surgery was not done, died of recurrence of bleeding in two weeks following the first episode. Patients having the more episodes in short period of time following the first hemorrhage show the poorer prognosis.

5) Specific angiographic findings, such as spasm, non-filling and mass lesion, high blood pressure above $160 \mathrm{~mm} \mathrm{Hg}$ and high CSF pressure above $250 \mathrm{~mm} \mathrm{H}_{2} \mathrm{O}$ were noted in majority of dead cases.

6) Fourteen were alert, three were confused, two were semicomatose and three were comatose before operation. All of the patients showing consciousness disturbance died and one out of 14 alert cases was lost postoperatively. Conservative treatment is preferable before improvement is obtained in consciousness state.

7) In eight cases, surgical intervention was done in one week following the last episode of hemorrhge and six out of them died. Surgery was made between one to three weeks following the last bleeding in five cases and one died postoperatively. Nine patients were operated upon after three weeks following the last accident and one was lost.

8) The reason why the result of the present report is not good comparing with other investigator's study, seems to be that many acute severe caces showing consciousness disturbance were present in the author's patients. 